\section{SCLC: Kraniale Radiatio verlängert Überleben}

Die prophylaktische Bestrahlung des Gehirns beim kleinzelligen Lungenkarzinom (SCLC) schien zwar vielversprechend - aber auch gefährlich. Daher lag die Methode lange auf Eis. Doch wenn die Bedingungen gut genug erforscht sind, scheint sie ihren Platz in der Therapie des SCLC zu haben.

V or knapp 40 Jahren erhielten die ersten Patienten mit SCLC eine prophylaktische Bestrahlung des Schädels (Prophylactic cranial irradiation, PCI), weil zahlreiche Chemotherapeutika die Blut-Hirn-Schranke nicht überwinden und im Gehirn deshalb besonders häufig Metastasen auftreten. Doch weil die Patienten Jahre nach der Therapie unter neurologischen Schäden litten, ließ der Enthusiasmus für die PCI erheblich nach. Inzwischen lässt sich die Methode recht sicher handhaben, wenn sie mit der Chemotherapie nicht zeitgleich, sondern sequenziell angewendet wird. Dennoch wird die PCI aufgrund von Sicherheitsund Wirksamkeitsbedenken bisher nicht universell eingesetzt.

Zur genaueren Abklärung von Nutzen und Risiken untersuchte eine Studie der
North Central Cancer Treatment Group nun das Schicksal von 739 SCLC-Patienten, die in vier verschiedenen Phase-IIund Phase-III-Studien eine PCI erhalten hatten. 318 Patienten litten unter ausgedehntem (ESCLC), 421 unter limitiertem SCLC (LSCLC). Alle wiesen nach Chemotherapie mit oder ohne Thoraxbestrahlung zur Zeit der PCI mindestens eine Krankheitsstabilisierung auf. 459 Patienten erhielten eine PCI nach einem der beiden Schemata $30 \mathrm{~Gy} / 15$ oder 25 Gy/10, 280 Patienten keine.

PCI-Patienten überlebten signifikant länger als Nicht-PCI-Patienten (Hazard Ratio [HR] 0,61). Nach einem Jahr lagen die Überlebensraten bei 56 bzw. $32 \%$, nach drei Jahren bei 18 bzw. $5 \%$. Die Signifikanz blieb auch bei stastischer Berücksichtigung von Alter, Performance- status, Geschlecht, Tumorstadium, Ansprechen und der Zahl der Metastasen bestehen (HR 0,82). PCI-Patienten hatten signifikant häufiger Nebenwirkungen vom Schweregrad $\geq 3(64 \%)$ als Nicht-PCI-Patienten $(50 \% ; p=0,0004)$, besonders oft litten sie an Alopezie und Lethargie. Die Dosisfraktionierung konnte nur für LSCLC-Patienten verglichen werden; dabei war die Dosierung $25 \mathrm{~Gy} / 10$ mit einem signifikant besseren Überleben assoziiert als $30 \mathrm{~Gy} / 15$ (HR $0,67 ; \mathrm{p}=0,018)$.

Fazit: Eine PCI bedeutet für Patienten mit ESCLC und LSCLC, deren Erkrankung nach Chemotherapie mit oder ohne Thoraxbestrahlung mindestens stabil ist, einen klaren Überlebensvorteil. Dabei ist die Art der Dosisfraktionierung von erheblicher Bedeutung. Allerdings kommt es unter der Behandlung auch zu zahlreichen schwerwiegenden Nebenwirkungen.

Christina Berndt

Schild SE et al. Prophylactic cranial irradiation in small-cell lung cancer: Findings from a North Central Cancer Treatment Group pooled analysis. Ann Oncol. 2012;23(11):2919-24.

\title{
Chemo-Immuntherapie des SCLC
}

\section{Bisherige Versuche, die Prognose beim fortgeschrittenen kleinzelligen Lungenkarzinom (SCLC) zu verbessern, verliefen enttäuschend. Die verschiedenen untersuchten Kombinationen auf Platinbasis konnten die Überlebenszeit nicht verlängern.}

n einer internationalen Phase-II-Studie wurde ergänzend zur Chemotherapie ein immunologischer Ansatz mit dem Anti-CTLA-4-Antikörper Ipilimumab untersucht. Die Substanz hat in ersten Untersuchungen bei verschiedenen Tumorentitäten ein dauerhaftes Ansprechen induziert; seit 2011 ist sie in Europa für die Behandlung des metastasierten malignen Melanoms zugelassen.

In die randomisierte, doppelblinde multizentrische Studie wurden 130 bisher unbehandelte SCLC-Patienten mit „extensive disease“ (ED) aufgenommen. Sie erhielten Carboplatin/Paclitaxel alleine oder mit zusätzlichem Ipilimumab, wobei dieses in einem Arm simultan, in einem anderen sequenziell nach der Che- motherapie gegeben wurde. Da sich der Verlauf unter Anwendung des speziellen Wirkprinzips - der Mobilisierung der körpereigenen Immunabwehr gegen den Tumor - mit den gebräuchlichen Kriterien nicht gut abbilden lässt, wurden in der Literatur vor Kurzem spezielle immunologische Ansprechkriterien, die immune-related response criteria (irRC), vorgeschlagen.

Unter dem schrittweise erweiterten Protokoll mit Ipilimumab verlängerte sich das immunologische progressionsfreie Überleben (Zeit bis zu einer $\geq$ 25\%igen Zunahme der Gesamt-Tumorlast oder Tod) gegenüber der ReferenzGruppe von median 5,3 auf 6,4 Monate (HR 0,64; $\mathrm{p}=0,03)$, nicht dagegen das progressionsfreie Überleben nach WHO-Kriterien. Die Gesamt-Überlebenszeit betrug in einer ersten Analyse 12,9 versus 9,9 Monaten; diese Differenz war nicht signifikant ( $\mathrm{p}=0,13)$.

Toxizitäten vom Grad 3 oder 4 kamen mit $43 \%$ resp. $50 \%$ deutlich häufiger in den Armen mit Immuntherapie vor als im Vergleichsarm (30\%). Dennoch waren die Abbruchraten vergleichbar.

Fazit: Ergebnisse, die unter Anwendung der neuen irRC-Kriterien gewonnen werden, erlauben keine Schlussfolgerungen, da letztere noch nicht validiert sind. Allenfalls können so Hypothesen entwickelt werden. Den Autoren zufolge rechtfertigen die Resultate aber eine weitere Erforschung der Ipilimumab-Anwendung beim SCLC. Waldtraud Paukstadt

Reck M et al. Ipilimumab in combination with paclitaxel and carboplatin as first-line therapy in extensive-disease-small-cell lung cancer: results from a randomized, double-blind, multicenter phase 2 trial. Ann Oncol. 2013;24(1):75-83. 